\title{
Energy and the Criteria for Radiation in General Relativity
}

\author{
R. ARnowitr* \\ Department of Physics, Syracuse University, Syracuse, New York \\ S. Deser $\dagger$ \\ Department of Physics, Brandeis University, Waltham, Massachusetts \\ AND \\ C. W. Misner $\ddagger$ \\ Universitetets Institut for Teoretisk Fysik, Copenhagen, Denmark
}

(Received December 3, 1959)

\begin{abstract}
The Hamiltonian for general relativity obtained in a previous paper furnishes a definition of energy whose physical interpretation is direct, and which fulfills the conditions required of the energy in other physical systems. The energy can be expressed as a surface integral at spacial infinity in terms of the spacial components of the covariant metric tensor at any given time. Thus, the energy depends only on the minimal initial Cauchy data and may be evaluated in any coordinate system, provided this system can be made asymptotically rectangular. These statements remain valid when particles are coupled to the gravitational field. The criteria for existence of gravitational radiation are formulated in terms of the canonical variables and the stress-tensor. These criteria are identical to those used in electromagnetic theory. Some applications are discussed.
\end{abstract}

\section{INTRODUCTION}

$I^{N}$ a previous paper, ${ }^{1}$ a canonical form for general relativity in terms of explicit canonical variables has been derived. These canonical variables were taken to be the two independent components of the transverse traceless parts of $g_{i j}$ and of

$$
\pi^{i j}=\left(-{ }^{4} g\right)^{\frac{1}{2}}\left(\Gamma_{m n}^{0}-g_{m n} \Gamma_{p q}^{0} \gamma^{p q}\right) \gamma^{i m} \gamma^{j n}
$$

i.e., $g_{i j}{ }^{T T}$ and $\pi^{i j T T}$. We have here made use of the general orthogonal breakup of a symmetric array $f_{i j}=f_{j i}$ :

$$
f_{i j}=f_{i j}{ }^{T T}+\frac{1}{2}\left[f^{T} \delta_{i j}-\left(1 / \nabla^{2}\right) f^{T}, i j\right]+f_{i, j}+f_{j, i} .
$$

In Eq. (1.2)

$$
\begin{aligned}
f^{T} & =f_{i j}-\left(1 / \nabla^{2}\right) f_{i j, i j}, \\
f_{i} & =\left(1 / \nabla^{2}\right)\left[f_{i j, j}-\frac{1}{2}\left(1 / \nabla^{2}\right) f_{j k, j k i}\right],
\end{aligned}
$$

while $1 / \nabla^{2}$ is the inverse of the flat space Laplacian operator (with appropriate boundary conditions) and $f_{i j}{ }^{T T}{ }_{, j}=0, f_{i i}{ }^{T T}=0$. This breakup is well defined on a space-like surface in terms of a given coordinate system. The specification employed here, as in III is

$$
\pi^{T}=0, \quad \frac{1}{2}\left(g_{i, j}+g_{j, i}\right)=\delta_{i j},
$$

* This research was supported in part by the U. S. Air Force under a contract monitored by The Aeronautical Research Laboratory, Wright Air Development Center.

$\dagger$ Supported in part by a National Science Foundation Research Grant.

$\ddagger$ Alfred P. Sloan Research Fellow. On leave from Palmer Physical Laboratory, Princeton University, Princeton, New Jersey.

1 The previous papers in this series will be referred to as I, II and III; they are: R. Arnowitt and S. Deser, Phys. Rev. 113, 745 (1959); R. Arnowitt, S. Deser, and C. W. Misner, Phys. Rev. 116, 1322 (1959), and Phys. Rev. 117, 1595 (1960). Notations and units are as in III, namely, $k=16 \pi \gamma c^{-4}=1, c=1$ where $\gamma$ is the Newtonian gravitational constant. Latin indices run from 1 to 3 , Greek from 0 to 3 , and $x^{0}=t$. All tensors and covariant operations and three-dimensional unless specified, e.g., $g^{i j}$ or $\gamma^{i j}$ is the matrix inverse to $g_{i j}$ and " $"$ " indicates covariant differentiation with respect to $g_{i j}\left(\right.$ not $\left.{ }^{4} g_{\mu \nu}\right)$. or alternately

$$
\pi^{i i}, j j-\pi^{i j}, i j=0, \quad g_{i j, j}=0 .
$$

The above coordinate conditions amount to using as independent variables in place of coordinates the metric functionals ${ }^{2} g_{i}=x^{i}$ and $\left(-1 / 2 \nabla^{2}\right) \pi^{T}=t$. It is, perhaps, important to realize, therefore, that this entire work involves only the functionals

and

$$
g_{i j}{ }^{T T}\left[g_{i},-\frac{1}{2}\left(1 / \nabla^{2}\right) \pi^{T}\right]
$$

$$
\pi^{i j T T}\left[g_{i},-\frac{1}{2}\left(1 / \nabla^{2}\right) \pi^{T}\right] \text {. }
$$

Such a situation is analogous to the statement of an orbit in ordinary particle mechanics in the form $r=r(\theta)$. Correspondingly our equations of motion for $g_{i j}{ }^{T T}$ and $\pi^{i j T T}$ are analogous to the orbit equations in terms of $d r / d \theta$. The same orbit, of course, can be described by way of the pair of equations $r=r(\tau)$ and $\theta=\theta(\tau)$ in terms of an arbitrary parameter $\tau$. While in particle mechanics there exist additional equations that allow one to determine the dependence of $\tau$ on the time $t$, in general relativity (due to the general covariance of the theory) there are no equations to determine the dependence of $g_{i}$ and $\left(1 / \nabla^{2}\right) \pi^{T}$ on the arbitrary coordinates $x^{\mu}$ that appear in the original action $\int d^{4} x\left(-{ }^{4} g\right)^{\frac{1}{2}}{ }^{4} R(x)$. Since these coordinates do not enter at any point in the canonical form of the theory, general covariance has been manifestly maintained and we are merely using the symbols $x^{\mu}$ as abbreviations for $g_{i}$ and $-\frac{1}{2}\left(1 / \nabla^{2}\right) \pi^{T}$.

It was shown in III that the components, $T^{0}{ }_{\mu}$, of the stress tensor took the form

$$
\begin{aligned}
& T^{0}{ }_{0}=-\mathcal{H C}=g^{T}, i i, \\
& T^{0}{ }_{i}=-2\left(\pi^{i},{ }_{j j}+\pi^{j},{ }_{i j}\right)=-2 \pi^{i i}{ }_{, j},
\end{aligned}
$$

${ }^{2}$ As was pointed out in III, the invariant functionals of the metric being used as coordinates take the form $g_{i}$ and $-\frac{1}{2}\left(1 / \nabla^{2}\right) \pi^{T}$ only in the preferred canonical frame of Eq. (1.4). In other frames these functionals take other forms to be obtained by making the appropriate coordinate transformations. 
where $\mathcal{H C}$ was the Hamiltonian density of theory. In these equations, $g^{T}{ }_{, i i}$ and $\pi^{i j}{ }_{, j}$ were to be expressed as functions of the canonical variables by solving for them in the constraint equations $G^{0}{ }_{\mu} \equiv R^{0}{ }_{\mu}-\frac{1}{2} \delta^{0}{ }_{\mu} R=0$. A perturbation solution of these equations, at least, clearly exists. Further, the coordinate conditions (1.4) ensure that $T^{0}{ }_{\mu}$ does not depend explicitly on $x^{\mu}$ as was shown in III. Thus, the standard conservation laws hold.

The energy-momentum of the field is just the volume integral of the components of $T^{0}{ }_{\mu}$ when a solution of the field equations is substituted in for $g^{T}$ and $\pi^{i}$. In this paper we will see how these expressions for energy and momentum may be evaluated without imposing the canonical coordinate conditions Eq. (1.4) or explicitly solving the constraint equations. With the aid of the expressions for energy and momentum we will be able to write down explicit criteria for the existence of gravitational radiation. In order to discuss radiation escaping to infinity we shall define a Poynting vector just as in electrodynamics. The condition for waves at a finite point is simply the nonvanishing of the canonical variables at this point.

The derivations of the canonical form and the definition of the energy-momentum of III were given only for the free gravitational field. In this paper we extend this analysis to include the problem of point particles coupled to the gravitational field. As will be seen, no essential changes are introduced by such a generalization. This extension allows us to examine the energy for some cases of interest. The possible relevance of these examples to the classical self-energy problem is discussed.

\section{DEFINITION AND PROPERTIES OF ENERGY AND MOMENTUM}

From Eq. (1.6) we see that the total energy may be written as

$$
E=-\int g^{T},{ }_{i i} d^{3} x=-\int g^{T}{ }_{i} d S_{i},
$$

where $d S_{1}=d x^{2} d x^{3}$, etc., are the rectangular surface elements at spacial infinity. Using Eq. (1.3a), the energy then becomes

$$
E=\int\left(g_{i j, j}-g_{j j, i}\right) d S_{i}
$$

Similarly, the momentum $P_{i}$ is given by

$$
P_{i}=-\int 2\left(\pi^{i},{ }_{j}+\pi^{j}, i\right) d S_{j}=-\int 2 \pi^{i j} d S_{j} .
$$

These equations have been derived in the canonical coordinate frame which by Eq. (1.4a) is asymptotically rectangular since $g_{i j}$ approaches $\delta_{i j}$ at infinity. In fact, as was shown in III, $g_{\mu \nu}$ approaches the rectangular Lorentz metric $\eta_{\mu \nu}$ at infinity. In this frame $g_{i j, j}$ vanishes. The utility of Eq. (2.2) is limited by the requirement that the integral be evaluated in the canonical frame. This restriction can be removed by the following argument. At spacial infinity, where the metric approaches the Lorentz value, coordinate transformations which preserve this boundary condition can be treated in the linear approximation. For the infinitesimal coordinate transformation, $\bar{x}^{\mu}=x^{\mu}+\xi^{\mu}, g_{i j}$ and $\pi^{i j}$ transform according to

$$
\begin{aligned}
& \bar{g}_{i j}=g_{i j}-\xi^{i}, j-\xi^{j}, i \\
& \bar{\pi}^{i j}=\pi^{i j}+\delta_{i j} \xi^{0}{ }_{, p p}-\xi^{0}{ }_{, i j} .
\end{aligned}
$$

As can be seen from Eqs. (1.3) or (1.2), $g^{T}$ and $\pi^{i}$ are invariant under this transformation. As a consequence, the energy and the momentum can actually be evaluated in any frame that is rectangular at infinity. ${ }^{3}$ The restriction to rectangular frames, conventionally used in all Lorentz covariant theories, can of course be removed by making use of standard flat space tensor analysis to calculate the energy in (asymptotically flat) spherical coordinates, for example.

Since the energy and momentum as given in Eqs. (2.2) are constant in time, they can be evaluated at any given time as in other dynamical systems. Thus, one should need, in order to calculate $P_{\mu}$, only those initial Cauchy data necessary to specify the state of the system uniquely. In general relativity, in the absence of coordinate conditions, these are $g_{i j}$ and $\pi^{i j}, 4,5$ and not for example $g_{0 \mu}$, which are needed only to describe how the coordinates are to be continued off the initial surface. As can be seen from Eq. (2.2), only $g_{i j}$ and $\pi^{i j}$ enter into the formulas for $P_{\mu}$. (In the canonical frame, only $g_{i j}{ }^{T T}$ and $\pi^{i j T T}$ are needed to specify the state of the system and these are, of course, sufficient for calculating the value of $P_{\mu}$.)

The value of the energy-momentum vector of Eqs. (2.2) can be shown to agree with those obtained from the surface integral forms derived from the Einstein pseudotensor, the Landau-Lifshitz pseudotensor, and Dirac's recent definition. ${ }^{6}$ This can be seen by linearizing the integrands in the surface integrals and noting that they reduce to Eqs. (2.2). Since the surface integrals are at spatial infinity, the linearization is rigorous.

\section{COUPLING WITH PARTICLES}

In the preceding discussion we have examined the properties of the energy and momentum of the uncoupled gravitational field. Since we shall be interested in solutions and problems involving sources, we shall

\footnotetext{
${ }^{3} \mathrm{~A}$ general test can be stated as to how fast the coordinate system must approach a rectangular one. One first calculates the functions $\xi^{\mu}$ according to $\xi^{i}=\bar{g}_{i}$ and $\xi^{0}=\frac{1}{2}\left(1 / \nabla^{2}\right) \bar{\pi}^{T}$. Two requirements must be imposed on $\xi^{\mu}$ in order that the statement that $g^{T}$ and $\pi^{i}$ are invariant be valid: First, the $\xi^{\mu}$ must vanish sufficiently rapidly that the quadratic terms neglected in Eq. (2.3) be negligible. Second, the terms on the right-hand side of Eq. (2.3) involving $\xi^{\mu}$ must vanish as rapidly as $g_{i j}-\delta_{i j}$ and $\pi^{i j}$.

${ }^{4}$ Y. Foures-Bruhat, J. Rational Mech. Anal. 4, 951 (1956)

${ }^{5}$ See also C. W. Misner and J. A. Wheeler, Ann. Phys. 2, 589595 (1957).

${ }^{6}$ P. A. M. Dirac, Phys. Rev. Letters 2, 368 (1959).
} 
here extend the analysis of the canonical formalism to the case of point particles coupled to the gravitational field. As will be seen, the coupling changes none of the formal results, and the expressions of Eq. (2.2) for energy and momentum now include the matter as well as the gravitational contributions.

The total action of the system is now

$$
I=\int{ }^{4} \mathfrak{g}^{\mu \nu}{ }^{4} R_{\mu \nu}\left(\Gamma^{\gamma}{ }_{\alpha \beta}\right) d^{4} x+\int L_{m} d^{4} x,
$$

where the matter Lagrangian density takes the form, ${ }^{7}$

$$
\begin{aligned}
& L_{m}(x)=\int d s\left\{p_{\mu}(s)\left[d x^{\mu}(s) / d s\right]\right. \\
& \left.\quad-\frac{1}{2} \lambda^{\prime}(s)\left[p_{\mu}(s) p_{\nu}(s){ }^{4} g^{\mu \nu}(x)+m_{0}{ }^{2}\right]\right\} \delta^{4}(x-x(s)) .
\end{aligned}
$$

We are considering here, for simplicity, the case of a single point particle with mass $m_{0}$. The matter Lagrangian is given in first order form where $p_{\mu}(s)$ and $x^{\mu}(s)$ are to be varied independently. In this parametric form of the matter action, the parameter $s$ is arbitrary (and not necessarily the proper time). The constraint equation,

$$
p_{\mu} p_{\nu}{ }^{4} g^{\mu \nu}(x(s))+m_{0}{ }^{2}=0,
$$

obtained by varying with respect to the Lagrange multiplier $\lambda^{\prime}(s)$ is, of course, the relativistic energymomentum relation for the particle. ${ }^{8}$

Varying $L_{m}$ with respect to $p_{\mu}(s)$, one obtains

$$
p_{\mu}(s)=\left[\left(1 / \lambda^{\prime}\right)\left(d x^{\nu} / d s\right)\right]^{4} g_{\nu \mu},
$$

which is the defining relation between the momentum and velocity. Note that only the combination $\lambda^{\prime} d s$ appears and there are no equations to determine $\lambda^{\prime}$ : Inserting Eq. (3.4) into Eq. (3.3) shows that a choice of $\lambda^{\prime}=1 / m_{0}$ corresponds to $s$ becoming the proper time. Finally, varying with respect to $x^{\mu}(s)$ gives rise to the usual geodesic equations of motion.

The arbitrary parameter $s$ could have been eliminated initially by performing the indicated $s$ integration in Eq. (3.2). The matter term in the action then becomes

$$
\begin{gathered}
I_{m}=\int d^{4} x\left\{p_{i} d x^{i}(t) / d t+p_{0}\right. \\
\left.-\frac{1}{2} \lambda\left[p_{\mu} p_{\nu}{ }^{4} g^{\mu \nu}(x)+m_{0}^{2}\right]\right\} \delta^{3}\left(x^{i}-x^{i}(t)\right), \\
\text { where } \quad \lambda(t)=\left[\lambda^{\prime}(s) d s / d x^{0}(s)\right]_{x^{0}(s)=t .}
\end{gathered}
$$

The solution of the constraint equation (3.3) is

$$
p_{0}=p_{i} \eta^{i}-N\left(m_{0}{ }^{2}+g^{i j} p_{i} p_{j}\right)^{\frac{1}{2}},
$$

where, as in III, $N=\left(-g^{00}\right)^{-\frac{1}{2}}, \eta_{i}={ }^{4} g_{0 i}$. Upon insertion of Eq. (3.7) into Eq. (3.5), the total action then takes

7 The four-dimensional $\delta$ function in Eq. (3.2) is defined according to $\int f(x) \delta^{4}(x-a) d^{4} x=f(a)$ for any scalar function $f(x)$. It thus transforms like a scalar density under coordinate transformations, but is not a functional of the metric.

8 Equation (3.3) may alternately be viewed as an example of Eq. (4.17) of II, $R=p_{n+1}+H=0$ in the discussion there of the parameter formalism. the form (to within a divergence)

$$
\begin{gathered}
I=\int d^{4} x\left\{\pi^{i j} \partial_{t} g_{i j}+p_{i}\left[d x^{i}(t) / d t\right] \delta^{3}\left(x^{k}-x^{k}(t)\right)\right. \\
+N\left[g^{-\frac{1}{2}}\left(g^{3} R+\frac{1}{2} \pi^{2}-\pi^{i j} \pi_{i j}\right)\right. \\
\left.-\delta^{3}\left(x^{k}-x^{k}(t)\right)\left(p_{i} p^{i}+m_{0}\right)^{\frac{1}{2}}\right] \\
\left.+\eta^{i}\left[2 \pi_{i}{ }^{j} \mid j+p_{i} \delta^{3}\left(x^{k}-x^{k}(t)\right)\right]\right\} .
\end{gathered}
$$

In Eq. (3.8), covariant notations are three-dimensional, ${ }^{1}$ e.g., $\pi=g_{i j} \pi^{i j}$. Varying Eq. (3.8) with respect to $N$ and $\eta^{i}$, one obtains the gravitational constraint equations,

$$
\begin{aligned}
g^{3} R+\frac{1}{2} \pi^{2}-\pi^{i j} \pi_{i j} & =\left({ }^{3} g\right)^{\frac{1}{2}} \delta^{3}\left(x^{k}-x^{k}(t)\right)\left(m_{0}{ }^{2}+p_{i} p^{i}\right)^{\frac{1}{3}}, \\
-2 \pi_{i}{ }^{j} \mid j & =p_{i} \delta^{3}\left(x^{k}-x^{k}(t)\right) .
\end{aligned}
$$

The total generator (obtained from variations at the endpoint, as in III) becomes

$$
G=p_{i}(t) \delta x^{i}(t)+\int d^{3} x\left[\pi^{i j} \delta g_{i j}+T^{0}{ }^{\prime} \delta x^{\mu}\right] .
$$

As in II, $T^{0}{ }_{\mu}{ }^{\prime}$ vanishes as a consequence of the con-

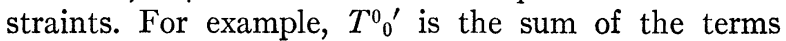
containing $N$ and $\eta^{i}$ in Eq. (3.8). If we now insert the constraint equations (3.9), the orthogonal decomposition (1.2) for $g_{i j}$ and $\pi^{i j}$, and the coordinate conditions $x^{i}=g_{i}$, and $t=-\frac{1}{2}\left(1 / \nabla^{2}\right) \pi^{T}$ into the generator Eq. (3.10), one obtains

$$
G=p_{i}(t) \delta x^{i}(t)+\int d^{3} x\left[\pi^{i j T T} \delta g_{i j}{ }^{T T}+T^{0}{ }_{\mu} \delta x^{\mu}\right],
$$

where

$$
\begin{aligned}
\mathcal{H}=-T^{0}{ }_{0} & =-g^{T},{ }_{i i}, \\
T^{0}{ }_{i} & =-2\left(\pi^{i},{ }_{j j}+\pi^{j},{ }_{, j}\right)=-2 \pi^{i j},{ }_{j} .
\end{aligned}
$$

In Eq. (3.12), $g^{T}$ and $\pi^{i}$ are to be expressed in terms of the canonical variables, $g_{i j}{ }^{T T}, \pi^{i j T T}, x^{i}(t), p_{i}(t)$, by solving the constraint equations (3.9). The generator (3.11) is now clearly in canonical form. Thus, $\mathcal{F C}$ is the Hamiltonian density of the entire coupled system and $T^{0}{ }_{i}$ is the total momentum density. The source on the right-hand side of Eq. (3.9) does not disturb the proof in III that $T^{0}{ }_{\mu}$ is independent of $x^{\mu}$. Thus, the conservation laws of the previous section still hold with the same coordinate conditions.

The energy $E$ of the coupled system becomes now

$$
E=-\int g^{T},{ }_{i i} d^{3} x=-\int g^{T},{ }_{i} d S_{i} .
$$

It would appear at first sight that the particle variables have disappeared in the expression for the total energy. However, if the energy is expressed in terms of canonical variables by solving Eq. (3.9) for $g^{T}, i i$, it is obvious that $x^{i}(t)$ and $p_{i}(t)$ appear. For example, to lowest order

$$
-g^{T},{ }_{, i i}=\mathcal{H C}_{L}+\left(m_{0}{ }^{2}+p_{i} p^{i}\right)^{\frac{1}{2}} \delta^{3}\left(x^{k}-x^{k}(t)\right),
$$

where $\mathfrak{F}_{L}$ is the linear theory Hamiltonian density,

$$
\mathfrak{F}_{L}=\frac{1}{4}\left(g_{i j}{ }^{T T}, k\right)^{2}+\left(\pi^{i j T T}\right)^{2} .
$$


The fact established here that the total energy for the full theory is expressible in terms of the asymptotic form of the metric is, of course, reasonable on physical grounds since it is the total energy of an isolated system (including all interaction energies, gravitational and others) which determines its Newtonian gravitational field at large distances. Note that the energy expression (3.13) still satisfies the basic requirement that it involve only initial Cauchy data [which are $g_{i j}, \pi^{i j}, x^{i}(t)$, and $p_{i}(t)$ at some fixed time in the absence of coordinate conditions, or $g_{i j}^{T T}, \pi^{i j T T}, x^{i}(t)$, and $p_{i}(t)$ in the canonical frame]. It does not depend on such coordinatesensitive quantities as $g_{0 \mu}$.

\section{APPLICATIONS}

As the simplest application of our energy formula Eq. (3.13) we examine the Schwarzschild solution. In isotropic coordinates (with the units we have been using ${ }^{1}$ ), the asymptotic form of the Schwarzschild solution becomes

$$
g_{i j}=\delta_{i j}+\delta_{i j} m / 8 \pi r .
$$

We now use the fact that for a tensor of the form $f_{i j}=\delta_{i j} f$, one has $f^{T}=2 f$. This result follows directly from Eq. (1.3a). Thus, at large distances

$$
g^{T}=m / 4 \pi r
$$

The energy, therefore, evaluates to $m$, as expected. The same result is obtained, of course, if one uses the asymptotic form of this metric in standard Schwarzschild coordinates:

$$
g_{i j}=\delta_{i j}+(m / 8 \pi r)\left(x^{i} x^{j} / r^{2}\right)
$$

Equation (4.2) is, in fact, more general then the simple Schwarzschild case; in fact, for any bounded system, the formula

$$
g^{T}=E / 4 \pi r+O\left(1 / r^{2}\right)
$$

holds. The equation $-g^{T}{ }_{, i i}=\mathcal{F C}$ has for its solution a multipole expansion where the coefficient of the monopole term is independent of angles, but possibly a function of time, i.e., $g^{T}=f(t) / 4 \pi r+O\left(1 / r^{2}\right)$. Equation (3.13) shows that $f(t)=E$ which is a constant in time.

For the Schwarzschild solution, which represents a static situation, one expects that there be no waves, that is, the gravitational canonical variables $g_{i j}{ }^{T T}$ and $\pi^{i j T T}$ (which can be initially specified independently of the particle variables) should vanish everywhere in the canonical frame. Indeed, $g_{i j}{ }^{T T}$ vanishes in any frame where $g_{i j}$ has spherical symmetry. This follows from the fact that there are no transverse vectors available, i.e., in Fourier space $g_{i j}$ can only depend on $\delta_{i j}$ and $k_{i} k_{j}$. Also, $\pi^{i j T T}$ vanishes due to spherical symmetry. One can show this explicitly for the canonical frame without actually transforming to it. ${ }^{9} \mathrm{We}$ write

\footnotetext{
${ }^{9}$ We have obtained the Schwarzschild solution in the canonical coordinate system and find that its metric components in this system involve quadratures that cannot be expressed in terms of standard functions. However, there exists another canonical frame in which the Schwarzschild metric takes on the usual isotropic form.
}

the general form for the spherically symmetric metric as

$$
g_{i j}=\delta_{i j}+f(r) \delta_{i j}+h(r) x^{i} x^{j} / r^{2},
$$

and impose the coordinate conditions of Eq. (1.4b), i.e., $g_{i j, j}=0$. The coordinate conditions lead to the equation

$$
f^{\prime}+h^{\prime}+(2 / r) h=0
$$

where $f^{\prime}=d f / d r$, etc. Since

$$
g_{i j}{ }^{T T}=g_{i j}-\delta_{i j}-\frac{1}{2}\left[g^{T} \delta_{i j}-\left(1 / \nabla^{2}\right) g^{T}, i j\right],
$$

in virtue of our coordinate conditions, one may compute easily that $g_{i j}{ }^{T T}$ vanishes if

$$
f^{\prime \prime}+h^{\prime \prime}-\left(f^{\prime}-h^{\prime}\right) / r-4 h / r^{2}=0 .
$$

Equation (4.6) and its first derivative are indeed equivalent to Eq. (4.8). Since this is a static metric, $\pi^{i j}$ vanishes; and, hence, the other coordinate condition of Eq. (1.4), $\pi^{T}=0$, is also satisfied. The conjugate momenta, $\pi^{i j T T}$, are similarly zero.

The fact that the canonical variables vanish in the canonical frame indicates that none of the energy resides in the independent modes of the gravitational field. This does not preclude the existence of static self-energies which are the nonlinear generalizations of the Coulomb type. For example, the quadratic terms of ${ }^{3} R$ from Eq. (3.9a) yields precisely the Newtonian self-interaction energy of a point particle,

$$
-\left(\gamma m_{0}^{2} / 2\right) \int d^{3} r \delta^{3}(\mathbf{r}) / r .
$$

To see how the self-energy arises in the full theory, we examine the constraint equation (3.9a), which becomes, for the static case,

$$
g^{\frac{1}{2} 3} R=m_{0} \delta^{3}(\mathbf{r}) \text {. }
$$

Note $m_{0}$ is the unrenormalized mass. Writing the metric in isotropic coordinates as ${ }^{\mathbf{1 0 , 5}} g_{i j}=[\chi(r)]^{4} \delta_{i j}$, Eq. (4.10) reduces to

$$
-8 \chi\left(\nabla^{2} \chi\right)=m_{0} \delta^{3}(\mathbf{r})
$$

which has the solution

$$
\chi=1+\left[m_{0} / \chi(0)\right][1 / 32 \pi r] .
$$

Since there is no energy in the independent modes of the gravitational field, we can identify the total energy as the renormalized mass $m$ of the particle

$$
E=m=m_{0} / \chi(0)
$$

The quantity $\chi(0)$, which may be obtained by considering Eq. (4.12) at $r=\epsilon$ (where $\epsilon \rightarrow 0$ ), is infinite:

$$
\chi(0)=\frac{1}{2}\left[1+\left(1+m_{0} / 8 \pi \epsilon\right)^{\frac{1}{2}}\right] .
$$

Thus, from Eq. (4.13), the total energy approaches zero as $\epsilon \rightarrow 0$, corresponding to a finite negative gravitational static self-energy $\Delta E=-m_{0}$. From the leading term of Eq. (4.13),

$$
E=\left(32 \pi m_{0} \epsilon\right)^{\frac{1}{2}}
$$

\footnotetext{
${ }^{10}$ A. Lichnerowicz, J. Math. pure appl. 23, 37 (1944).
} 
we see that the total energy vanishes as $\epsilon^{\frac{1}{2}}$ rather than diverging as $-\epsilon^{-1}$ (the Newtonian result). Of course, a full discussion of the self-energy must wait until dynamical effects are included.

\section{RADIATION CRITERIA}

Having put general relativity into canonical form, we have thus separated out those gravitational field variables of the theory which are independent of the source variables. Excitations in such independent variables provide a primary definition of what one calls waves or radiation. This is, of course, the same definition for radiation as that given in electrodynamics or any other field theory. This viewpoint is taken as a matter of course in those field theories where no redundant variables appear. In electrodynamics, the gauge invariance obscures to some extent the fact that it is only transverse modes of the vector potential and electric field that need be examined to recognize radiation. These variables are just the canonical ones of the Maxwell field. Correspondingly in general relativity, the basic requirement for the existence of radiation is to be formulated solely in terms of the canonical variables. Stated formally: The nonvanishing of $g_{i j}{ }^{T T}$ or $\pi^{i j T T}$ at a point in the canonical coordinate system represents the existence of a wave carrying energy and momentum. As in electrodynamics, radiation and induction effects can be meaningfully separated only in the "wave zone", but also as in electrodynamics the above criteria can in fact be employed consistently nearer the sources.

Aside from these requirements, which apply locally or for bounded systems, one can also formulate requirements for the situation of radiation escaping to infinity. Again as in electrodynamics, one simply examines the Poynting vector $T^{i 0}$ at infinity; i.e., $T^{i 0} d S_{i}$ represents the flux of energy through the two-dimensional surface elements $d S_{i}$ at infinity. There, $T^{i 0}$ takes on its linearized form, since at infinity this weak field form is rigorous. The symmetric $T^{\mu \nu}$ for the linearized theory was given in I. By direct calculation one finds that this

$$
T^{i 0} \text { is } 2 \pi^{l m T T}\left(\Gamma^{i}{ }_{l m}\right)^{T T},
$$

where

$$
\left(\Gamma^{i}{ }_{l m}\right)^{T T}=\frac{1}{2}\left(g_{l i}{ }^{T T}{ }_{, m}+g_{m i}{ }^{T T}, l-g_{l m}{ }^{T T}{ }_{, i}\right) .
$$

This is identical to the leading term of $-2 \pi^{i j},{ }_{j}$ obtained by solving the constraint equation

$$
-2 \pi^{i j}{ }_{1 j} \equiv-2 \pi^{i j}, j-2 \pi^{l m} \Gamma^{i}{ }_{l m}=0 .
$$

In the canonical frame then, the Poynting vector at infinity is

$$
T^{i 0}=-2 \pi_{, j}^{i j}=2 \pi^{l m T T}\left(\Gamma_{l m}^{i}\right)^{T T} .
$$

If other systems are coupled to the gravitational field, $-2 \pi^{i j}{ }_{j}$ now represents the total energy flux at infinity, of which the purely gravitational part is the last member of Eq. (5.2). The point to be stressed here is that once general relativity has been put into canonical form, the physical interpretations to be given to radiation are identical to those of other field theories. Within this framework, one should be able to deal meaningfully also with idealized situations, such as cylindrical waves and plane waves.

\section{DISCUSSION}

In this paper we have examined some of the physical quantities that arise when one has cast general relativity into canonical form. In particular, we have defined the energy and momentum of the field, which are still the basic integrals needed to characterize the system independently of its internal structure. Both these quantities can be expressed in terms of the canonical variables of the theory and hence can be determined from minimal initial Cauchy data.

It is a noteworthy physical property of general relativity that the total energy and momentum can be expressed as surface integrals. This finds its analogy in electromagnetism where the source of that field, namely, the total charge, may be characterized in the same way. For the coupled system we have seen that the excitations of the gravitational field contribute to the total energy of the system seen in the asymptotic Newtonian potential (along with the matter and interaction energies). Further, the coupling of matter does not affect the definition of the canonical variables of the gravitational field.

The highly nonlinear fashion in which the matter interacts with the gravitational field in even the simplest case was illustrated in the treatment of the static point particle. Here it was seen that the usual Schwarzschild mass parameter was really a renormalized mass. While to lowest order in a perturbation expansion in the gravitational coupling constant, the self-energy was seen to be the linearly divergent Newtonian term, the rigorous static self-energy was found to cancel the unrenormalized mass with the total energy vanishing as a square root. ${ }^{11}$

The detailed properties of the gravitational field are determined by an examination of the excitation present in the canonical modes. Thus, criteria for radiation can be stated in these terms. For example, to interpret the total energy of the Schwarzschild field as the renormalized mass of a single particle, it was necessary to establish that none of the independent gravitational modes were excited. Alternately this fact is what permits one to say that the solution represents a "one-particle" state in the field-theoretical sense.

The results of this paper have been derived using a particular set of coordinate conditions with associated canonical variables. Actually, all results are independent of the choice of canonical frame. This will be demonstrated in a later paper.

\footnotetext{
${ }^{11}$ Further results on the classical self-energy problem for neutral and charged particles are given in Phys. Rev. Letters 4, 375 (1960).
} 